\title{
IMPROVING THE ASSESSMENT OF CHILDREN AND YOUTH WHO PRESENT WITH GENDER DYSPHORIA
}

\author{
Kathleen Walsh ${ }^{1}$, Melissa Jonnson ${ }^{2}$, Wallace Wong ${ }^{3}$, \& Veronique Nguy ${ }^{4}$ \\ ${ }^{1,4}$ University of British Columbia, (Canada) \\ ${ }^{2}$ Simon Fraser University (Canada) \\ ${ }^{3}$ Diversity \& Emotional Wellness Centre (Canada)
}

\begin{abstract}
Practitioners working with gender non-conforming children and youth ascribe to general guidelines based on the World Professional Association for Transgender Health Standards of Care for the Health of Transsexual, Transgender, and Gender Nonconforming People (2012). Guidelines inform clinical practice and assessment and emphasize the need for gender affirming care but do not include strict treatment criteria. Consequently, there are multiple perspectives and approaches in the field regarding effective assessment and treatment of gender diverse and transgender clients. The current study investigates the perspectives and satisfaction of transgender youth and their parents surrounding gender health assessments (e.g., hormone readiness assessments). Twenty-five parents and 22 youth who were accessing gender health services through a community outpatient clinic completed a questionnaire about the assessment process. Data was analyzed using mixed methods. Similar response patterns were found between groups. When asked about the need for comprehensive assessment $(92.0 \% ; n=23)$ of parents and $(91.0 \% ; n=20)$ of youth agreed or strongly agreed that a comprehensive assessment is necessary. Emergent themes included the use of comprehensive assessment as a measure to rule out other contributing factors, to increase understanding and support for gender variant expression, and to aid in identity formation. Regarding the number of sessions needed to complete a comprehensive assessment, parents and youth shared similar perspectives with the mean number of $3.96(S D=0.96)$ desired sessions for parents and 3.14 sessions $(S D=1.14)$ for youth. Qualitatively, the most common theme shared by parents and youth was the importance of taking an individualized approach to assessment length depending on the needs of the child and family. The greatest variation between response groups was in reference to whether parents should be involved in the assessment process. Among parents, almost all $(92.0 \% ; n=23)$ agreed or strongly agreed that parents should be involved in the process, whereas just over half of youth $(63.6 \% ; n=14)$ felt this way. Additionally, $36.4 \%(n=8)$ of youth disagreed or strongly disagreed with parental involvement. Rationale for involvement included increasing parents' understanding and support of the child and being able to provide important details about development. Many youth shared comments about the complexity of parental support and how involvement must depend on the level of support from the parents and their relationship with their child. This study aims to increase clinical understanding of the experiences of those seeking gender health assessment services to inform and improve practices to better serve this community.
\end{abstract}

Keywords: Gender dysphoria, comprehensive assessment, children \& youth, transgender, cross gender hormone treatment.

\section{Introduction}

Currently in North America there is a trend for transgender youth to access gender-related health care and interventions based on the Informed Consent Model (Edwards-Leeper, Leibowitz \& Sangganjanavanich, 2016). The consent model allows transgender clients, typically adults, to access treatment and interventions including hormone treatment and surgery without receiving a comprehensive mental health evaluation (Schulz, 2018). The Consent Model offers a more accessible, affirmative, timely and less restrictive treatment model for transgender individuals, but questions remain about its efficacy (Edwards-Leeper et al., 2016; Schulz, 2018). This approach continues to grow in popularity among adult clients and many practitioners; however, when applied to younger clients, debate remains. Children and youth have complex needs and are significantly more reliant on outside support systems for meeting their 
needs including familial support, financial assistance, psychological support, housing, transportation and more (Edwards-Leeper et al., 2016).

Alternatively, comprehensive psychological assessments, or the current standards of care, for assessing Gender Dysphoria require clinicians to assess the mental health and well-being of the patient seeking care, in addition to having the opportunity to assess the socio-familial factors that are central to successful treatment implementation and adherence (World Professional Association for Transgender Health [WPATH], 2012). This approach is supported by such organizations as the American Psychological Association (APA, 2015), the Australian and New Zealand Professional Association for Transgender Health (ANZPATH, 2019) and the Royal College of Psychiatrists (2014). While more thorough than the informed consent model, there are both time-related and financial costs associated with the comprehensive approach. In addition, including a mental health assessment in the comprehensive assessment could contribute further to the pathologization of gender variance.

Given that the WPATH Standards of Care for the Health of Transsexual, Transgender, and Gender Nonconforming People (2012) do not have specific guidelines for the assessment of children and/or youth presenting with gender dysphoria, clinicians determine a course of treatment based on their training, comfort zone and previous experiences (Edwards-Leeper et al., 2016). Therefore, there is little consistency across professionals when supporting children and youth with gender variance. Often, clinicians feel pressure to act quickly, especially if the child/youth is dysphoric and presents with severe mental health challenges including self-harm and suicidal behaviours. While acting quickly may be intended as an act of support for the child/youth, it could be short-sighted by failing to consider the complexities of the age group and the long-term developmental, social, cultural and financial needs of the child/youth. For instance, a youth may be provided with a prescription for gender affirming hormones but be denied the financial and practical support necessary to access the treatment by their parents, which could trigger an exacerbation of prior mental health concerns. As such, comprehensive assessments that involve a parent or other supportive adult may result in greater long-term success and safety for the youth.

The ongoing debate among mental health and medical professionals involves a fine balance between the important facilitation of client access to services and consideration of the potential complicating factors previously mentioned. This study aims to increase clinical understanding of the experiences of those seeking gender health assessment services, both youth and parents of gender variant children, to inform and improve current practices in order to better serve this community.

\section{Participants and methods}

Forty-eight people participated in this study and were recruited through a community-based mental health service clinic in British Columbia, Canada. Participants were divided into two groups: 22 gender variant youth ranging from 13 to 19 years $(M=16 ; S D=1.95)$ and 25 parents of gender variant children/youth ranging in age from 8 to 19 years $(M=12.76 ; S D=2.60)$. Of the youth participants, 17 reported their affirmed gender as male, 2 as female, 1 as non-binary, 1 as questioning and 1 did not report. Of the parent respondents, 15 reported their child's affirmed gender as male, 6 as female, 1 as gender fluid and 3 as non-binary. Of all respondents, 25 had previously undergone a comprehensive gender assessment, 16 had not, 3 were in progress of getting an assessment and 4 did not report if they had had an assessment or not. After completing appropriate informed consent procedures, participants completed a three-item questionnaire. Items queried participants' perspectives about (1) the need for a comprehensive assessment when a youth presents with gender identity concerns, (2) the number of sessions needed to complete a comprehensive assessment, and (3) the importance of parents being involved in the assessment process. For each item, a categorical response option (e.g., strongly disagree, disagree, neutral, agree, strongly agree) was provided, in addition to an open-ended option. Frequency tables were analyzed to determine differences in responding between the two groups, and open-ended responses were analyzed for emergent themes. Exemplar quotes are shared to help illustrate each theme.

\section{Results}

Preliminary results of this ongoing study are provided below. Analyses are divided into three sections based on the questionnaire items, and emergent themes are explored within each item's responses. 


\subsection{Necessity of a comprehensive assessment}

Item 1 asked participants the extent to which they agreed that a comprehensive assessment is needed when a child or youth presents with gender identity concerns. Patterns of responding were similar across groups with almost all parents $(92.0 \% ; n=23)$ and youth $(91.0 \% ; n=20)$ agreeing or strongly agreeing that a comprehensive assessment is necessary. Of the remaining participants, one parent and one youth strongly disagreed and one parent and one youth provided a neutral response. Open-ended responses among parents highlighted the importance of the assessment for recommendations on how to best support their child. One parent wrote, "The report that came from the assessment has helped my child. It is part of their school file and the recommendations have been used as a guide for sports/classes. The assessment is fine but is all things us (parents) knew already." Another parent shared "We did not know about the details of transitioning until we did the assessment. I feel better as a parent about treatments having gone through the assessment process."

Common themes among both groups included the importance of the assessment for ruling out underlying and contributing factors, as well as the importance of the assessment for identity formation. One parent shared that it "helps to gain clarity of where your child's emotions and feelings are coming from - if there is anything underlying (contributing factors), [to] gain an understanding of [the] child's self-perception, [and to] give [the] child an opportunity and voice to share and hopefully make sense of their feelings and self-perception." One youth shared that "I think that it is very important that a child gets to learn about themselves through an assessment like this one. As long as the concerns are great enough, then it would be a good idea." Another youth expressed that "[an assessment] can isolate other factors that may give them gender conflictions (mostly in young children). I think most teenagers are mature and independent enough to decide their gender for themselves." Some youth also shared a more reserved perspective indicating that an assessment is "not initially [necessary]. One should have their own time to figure things out rather than be bombarded with questions."

\subsection{Number of sessions needed}

Item 2 asked participants how many sessions would be adequate to complete a comprehensive gender related assessment. Response options included a half hour appointment, 1 session, 2 sessions, 3 sessions, 4 sessions and 'other'. On average, parents $(M=3.96 ; S D=0.96)$ and youth $(M=3.14$; $S D=1.14$ ) reported that a similar number of sessions would be necessary. Parents' open-ended responses reflected the idea that the assessment length should be determined by the professional and based on the individual circumstances and needs of the child and their family. One parent noted that "this should be left up to the professional. Each child is unique and a 'cookie cutter' approach does not acknowledge this. [The] age of [the] child, past history, [and] current life circumstances all impact willingness of [the] child and family to disclose what is happening." Similarly, several youth pointed out that the age of the client may impact the number of sessions needed. For example, one youth shared, "I was 18 and felt that 2 sessions was enough, but I was also sure how I wanted to pursue. I think younger people, or those who are unsure, should have longer to explore in sessions."

\subsection{Parental involvement in the assessment}

Item 3 asked participants the extent to which they agreed that parents/caregivers should be involved in the assessment process. Among parents, almost all $(92.0 \% ; n=23)$ agreed or strongly agreed that parents should be involved in the process. Of the two remaining parents, one was neutral, and one strongly disagreed. Conversely, there was much more variability among youths' responses to this question. Over half of youth $(63.6 \% ; n=14)$ agreed or strongly agreed in favor of parent involvement, whereas $36.4 \%(n=8)$ disagreed or strongly disagreed. In addition to these figures, the themes that emerged through open-ended responses provide important detail to conceptualize this data.

The most prominent theme that emerged from parents' open-ended responses included that the assessment process helps to increase parent's understanding and support of their child. One parent wrote that "the entire family is transitioning and learning. The youth needs the support of their parents and family." Another wrote that the assessment provides "an opportunity for parents to learn about this aspect of their child - an opportunity for improved communication and understanding between parents and child." The second most prominent theme that emerged from parent responses was that parents can provide unique knowledge about their child that is important for the integrity of the assessment. For example, one parent wrote that "parents are an important part of a child's life and often know much of what the child is going through."

Alternatively, the most prominent theme that emerged from youths`open-ended responses was the complexity of parental support. Almost unanimously, youth expressed that if parents are supportive of their child's gender identity journey then parental involvement is generally beneficial. One youth wrote that "I believe it truly depends. If parents are accepting and supportive - yes. If they want to, they should, 
unless the child doesn't want them to." Alternatively, concerns about the impact of parental involvement were raised. One youth wrote that "I don't think parents should be involved during the assessment because they can limit the openness and safety felt by the child." Notably, although a similar theme arose in the parent data, only three parent respondents raised this concern. Another prominent theme that emerged for youth was the beneficial impact the assessment can have on parents insight and ability to effectively support their child. This theme is similar to the one that emerged among parents as previously discussed. For instance, one youth highlighted that "the opportunity for parents to be present could be seen as beneficial towards their learning of their child's feelings and the subject."

Other less common themes across both groups included the importance of a child-focused assessment, regardless of parental involvement and the child's dependence on the parent to help direct the assessment process and implement recommendations. Parental dependence was noted to be particularly important for younger children.

\section{Discussion}

Results of this research revealed several shared perspectives between parents and youth regarding gender health assessments. Commonalities were found in regards to the importance of having a comprehensive assessment, as well as the length (i.e., number of sessions) needed. However, differing perspectives were noted in regard to parental involvement in the assessment process. Parental involvement was a polarizing topic for many youth, and responses revealed the complex nature of parental support. Alternatively, parents were almost unanimously in favor of parental involvement.

Parents play a central role in the lives of youth. Financial, logistical, social/emotional and physical support are just some ways in which parents may support their children. Despite the capacity of some youth to legally consent to the assessment, in the vast majority of cases, it is not an ideal situation for a clinician to complete an assessment without completing a thorough evaluation of these areas of support and at least make attempts to engage these external support systems. At the same time, it may be necessary to consider other sources of support (e.g., other trustworthy adults) if parents are unsupportive of their child's needs. Relatedly, youth and parents in this study emphasized the importance of taking an individualized approach to assessment. Since there is no standardized, universally-accepted approach for conducting a gender health assessment, clinicians are responsible for considering all relevant familial, developmental, cultural, psychological, and logistical factors impacting clients with gender health concerns. A thorough consideration of these factors will not only help professionals facilitate youths' access to treatment but also increase the likelihood that their clients will successfully adhere to treatment and access support throughout this often challenging process.

\subsection{Limitations}

Primary limitations of this research include the homogenous nature of recruiting all participants through a community-based mental health office. All participants were affiliated with this office due to previously showing interest in pursuing an assessment and/or receiving some type of mental health support for gender health concerns. Additionally, the team behind this research includes clinicians from a specialized Gender Health Program, thus adding an additional confound. Another limitation is the moderate sample size of the study. It is hard to make gross generalizations given the sample size and heterogeneity of the sample. That being said, the sample size is relatively large given the specificity of the topic and persons being researched.

\section{Conclusion}

Findings from both youth and parents indicate strong agreement with the need for a comprehensive gender health assessment with youth present with concerns about their gender identity. Both groups identified that a thorough and individualized comprehensive assessment can help parents better understand and support their children's overall needs, in addition to their gender-related needs. Additionally, a comprehensive assessment was noted to provide families with a clearer understanding of current and future transitions and challenges people may face along their gender identity journey. Both groups also reported that having multiple sessions was necessary for completing a comprehensive assessment given the complexity and uniqueness of each client. Having multiple sessions provides an opportunity for parents and youth to become more engaged in the process and provides opportunities for families to enhance their mutual communication and support. 
Results also indicate that parents and youth have differing perspectives about parental involvement in the assessment process. While most youth see the benefit of having their parents involved, this involvement was dependent on whether the parents were perceived as supportive or unsupportive of their child's gender identity and journey. Many youth expressed caution about the motives of some parents in interfering with their child's progress and transition. While it is reasonable for youth to hold this view, it is also important to acknowledge that many parents are new to the gender assessment process, in comparison to their child. Parents are typically seeking more information to better understand what their child is going through and are sometimes resistant to the process due to a desire to protect their child. A lot of misinformation exists regarding gender variance, identity and expression, and parents may require professional guidance to support them in understanding what their child is going through. Arguably, for this reason, having parents involved may be even more crucial in helping to clarify misconceptions thus allowing them to provide more informed, proactive support for their child.

\section{References}

American Psychological Association (2015). Guidelines for psychological practice with transgender and gender nonconforming people. American Psychologist, 70(9), 832-864. doi:10.1037/a0039906

Coleman, E., Bockting, W., Botzer, M., Cohen-Kettenis, P., DeCuypere, G., Feldman, J., Zucker, K. (2012). Standards of care for the health of transsexual, transgender, and gender-nonconforming people, version 7. International Journal of Transgenderism, 13(4), 165-232. doi:10.1080/155 32739.2011.700873

Edwards-Leeper, L., Leibowitz, S., \& Sangganjanavanich, V. F. (2016). Affirmative practice with transgender and gender nonconforming youth: expanding the model. Psychology of Sexual Orientation and Gender Diversity, 3(2), 165-172. doi:10.1037/sgd0000167

Schulz, S. L. (2018). The informed consent model of transgender care: An alternative to the diagnosis of gender dysphoria. Journal of Humanistic Psychology, 58(1), 72-92. doi:10.1177/0022167 817745217

Telfer, M. M., Tollit, M. A., Pace, C. C., \& Pang, K. C. (2018). Australian standards of care and treatment guidelines for transgender and gender diverse children and adolescents. Medical Journal of Australia, 209(3), 132-136. doi:10.5694/mja17.01044

World Professional Association for Transgender Health. (2012). Standards of care for the health of transsexual, transgender, and gender-nonconforming people, 7th version. Retrieved from https://amo_hub_content.s3.amazonaws.com/Association140/files/Standards\%20of\%20Care, \%20 V7\%20Full\%20Book.pdf.

Wylie, K., Barrett, J., Besser, M., Bouman, W. P., Bridgman, M., Clayton, A., . . Rathbone, M. (2014). Good practice guidelines for the assessment and treatment of adults with gender dysphoria. Sexual and Relationship Therapy, 29(2), 154-214. doi:10.1080/14681994.2014.883353 